\title{
High-resolution vertical imaging of the troposphere and lower stratosphere using the new MU radar system
}

\author{
H. Luce ${ }^{1}$, G. Hassenpflug ${ }^{2}$, M. Yamamoto ${ }^{2}$, and S. Fukao ${ }^{2}$ \\ ${ }^{1}$ Laboratoire de Sondages Electromagnétiques de l'Environnement Terrestre, Université de Toulon et du Var, CNRS, La \\ Garde, 83957, France \\ ${ }^{2}$ Research Institute for Sustainable Humanosphere, Kyoto University, Uji, 611-0011, Japan
}

Received: 29 July 2005 - Revised: 16 February 2006 - Accepted: 28 February 2006 - Published: 19 May 2006

\begin{abstract}
In the present paper, a new application of the range imaging technique called Frequency Interferometry Imaging (FII) or Range Imaging (RIM), performed in April 2005, is shown using the new 46.5-MHz Middle and Upper (MU) atmosphere radar system (Shigaraki, Japan). Heighttime images of brightness distribution have been computed at the highest resolution ever obtained for imaging with VHF radars in the troposphere and, for the very first time, in the lower stratosphere, up to about $22 \mathrm{~km}$. The images were produced by processing signals obtained with an initial rangeresolution of $\Delta r=150 \mathrm{~m}$ and five equally-spaced frequencies within $\Delta f=1.0 \mathrm{MHz}$, with the adaptive Capon method. These values represent an improvement of a factor 2 over all the previous published experiments at VHF, which were performed with $\Delta r=300 \mathrm{~m}$ and $\Delta f=0.5 \mathrm{MHz}$. The Capon images present realistic and self-consistent features, and reveal many more organized structures than the height-time SNR plots at the initial range-resolution. For example, the Capon images show persistent enhanced brightness layers significantly thinner than $150 \mathrm{~m}$ in the stratosphere, which are impossible to track with the standard single-frequency mode owing to a lack of range resolution. These observations thus support the idea of strong stratification even at vertical scales much smaller than $100 \mathrm{~m}$, as suggested by recent highresolution temperature observations by balloons (Dalaudier et al., 1994). We also present comparisons of Capon images with patterns obtained from the dual-FDI technique and two parametric methods (the MUSIC algorithm and the newlyintroduced Maximum Entropy Method based on an autoregressive (AR) model). The comparisons confirm the insufficiencies of the dual-FDI technique and indicate that parametric methods such as MEM and the MUSIC algorithm can
\end{abstract}

Correspondence to: $\mathrm{H}$. Luce

(hubert.luce@1seet.univ-tln.fr) help to validate the Capon images when the parametric methods provide similar patterns.

Keywords. Radio science (Interferometry) - Meteorology and atmospheric dynamics (Turbulence) - Electromagnetics (Signal processing and adaptive antennas)

\section{Introduction}

This work describes an application of range imaging (called RIM by Palmer et al., 1999, or FII, Frequency Interferometry Imaging, by Luce et al., 2001a) with the new multi-frequency digital multi-receiver system of the 46.5-MHz MU radar operational since March 2004. In previous papers, some authors have demonstrated by both simulations and applications the potential of FII (e.g. Palmer et al., 1999, 2001; Luce et al., 2001a; Smaïni et al., 2002; Chilson et al., 2001; Fernandez et al., 2003; Yu and Brown, 2004) for improving the resolving performance of Stratosphere-Troposphere (ST) radars, which are limited by their receiver bandwidth, and for eliminating the drawbacks of the dual-FDI (Frequency Domain Interferometry) technique (e.g. Luce et al., 2001c). These efforts are important for a better description of the thin turbulent layers and temperature gradient sheets in the stratified atmosphere from VHF ST radar measurements, since the ST radar technique is still one of the most important sources of information about the dynamics and structure of the upper troposphere and lower stratosphere.

Other techniques than FII have been proposed to mitigate the bandwidth limitations of MST radars. For example, Röttger and Schmidt (1979) proposed, very early on after the development of these radars, a method based on the reduction of the original sampling time interval by shifting the samples and a special deconvolution method by means

Published by Copernicus GmbH on behalf of the European Geosciences Union. 
of contrast enhancement, as used in optics. Also, very recently, Zhang et al. (2005) applied on a weather radar a technique which uses the fact that the cross-correlation function of signals from two overlapped resolution volumes results from scatterers in the common volume.

Preliminary results of FII observations using only limited data sets with the old MUR were presented by Luce et al. (2001a, b). The technical configuration was not adapted to optimal applications, since only switches for two frequencies were available. Sequential series of observations in dualFDI observational mode with different frequency spacings were used, likely producing large uncertainties regarding the computed parameters and errors. Subsequently, a temporary system using four frequency synthesizers with a maximal frequency excursion of $0.5 \mathrm{MHz}$ switched pulse-to-pulse was then developed and the results have been described by M. Hirono (2003, Master Thesis, personal communication). In contrast, the recently upgraded system can provide observations with a sequential pulse-to-pulse 5-frequency scanning from 46.0 to $47.0 \mathrm{MHz}(\Delta f=1.0 \mathrm{MHz})$, with an initial range-resolution of $\Delta r=1 / 2 c \tau=150 \mathrm{~m}$, where $\tau$ is the pulse duration, and $c$ is the speed of light. It is thus improved by a factor of 2 with respect to all existing experiments in frequency domain interferometric modes (including both dualFDI and FII) published until now with VHF ST radars, since $\Delta f=0.5 \mathrm{MHz}$ and $\Delta r=300 \mathrm{~m}$ (i.e. $\tau=2 \mu$ s) were usually used. It has to be noted that for $n$ equally-spaced frequencies, the maximum permissible $\Delta f(\mathrm{MHz})$ value in order to avoid ambiguity lobe effects is $(n-1) / \tau$ (with $\tau$ in $\mu$ s) and not $1 / \tau$, as seems to be the case from the numerical values used. However, in practice, the values of $\Delta f$ and $\tau$ are limited by the receiver bandwidth and frequency band allocations. For better resolution performance, it would better to use $\Delta f>1 / \tau$ (to the detriment of ambiguity lobe rejection) when possible. Chilson et al. (2003) used $\Delta f=2 \mathrm{MHz}$ and $\tau=2 \mu$ s with a UHF radar, while Smaïni et al. (2002) used $\Delta f=0.75 \mathrm{MHz}$ and $\tau=2 \mu$ s with a mini VHF radar.

In Sect. 2, a brief description of the principles of the FII technique, radar configuration and data processing used is given. In Sect. 3.1, results of observations from $4.35 \mathrm{~km}$ up to $22.20 \mathrm{~km}$, using the Capon method (e.g. Palmer et al., 1999; Luce et al. 2001a) are described and compared with the results from the standard single-frequency observational mode. A portion of the Capon images is then described in more detail in Sect. 3.2 and compared with the patterns obtained from the MUSIC algorithm (Luce et al., 2001a), the newlyintroduced auto-regressive (AR) Maximum Entropy Method (MEM) and the dual-FDI technique (Kudeki and Stitt, 1987). Section 3.3 presents an attempt at estimation of the layer thickness from the Capon method and the results are compared with the thickness provided by the dual-FDI technique. Finally, conclusions of this work are given in Sect. 4.

\section{Generalities}

\subsection{Principles of the FII technique}

The principles of range imaging have been described in detail by previous authors (e.g. Palmer et al., 1999; Luce et al. 2001a). It can be simply recalled that range imaging is similar to antenna array processing used for carrying out angular scanning. Range imaging provides the distribution of backscattered power (called "brightness distribution") as a function of altitude. Range imaging needs to estimate first the covariance matrix of received signals at all possible different frequency spacings. The brightness distribution obtained by performing scanning in a given radar range gate can differ according to the processing methods used. Basically, two classes of methods can be applied. First, there are the (non-parametric) filter-bank methods, such as the Capon method, which do not require hypotheses on the vertical distribution of the scatterers. The Capon method is an adaptive method which provides a better resolution than the Fourier method when the signal-to-noise ratio (SNR) is sufficient (e.g. Palmer et al., 1999). This method is well-recognized and has been successfully applied, for example, by Chilson et al. (2003) on a UHF profiler.

The second class of methods includes parametric methods based on a selected model. Among these methods, the MUSIC algorithm is based on the singular value decomposition (SVD) of the covariance matrix. It has been developed for the optimal detection of discrete non-coherent sources and its performance does not depend on SNR. The MUSIC algorithm does not provide quantitative information on the intensity of the assumed discrete sources, since it is equivalent to Capon processing when SNR tends to infinity (e.g. Nickel, 1988). Thus, when sources (here, atmospheric layers) can be considered as "thin enough when compared to the range resolution", SVD-based methods are optimal and can provide much better resolution than filter bank-methods (e.g. Luce et al., 2001a).

The Maximum Entropy Method (MEM) is another parametric method originally developed in spectral analysis for the detection of narrow-band spectra with a low-order autoregressive (AR) model (e.g. Stoïca and Moses, 1997). Hence, for our application, MEM would also provide a better resolution than the Capon method in the case of thin atmospheric layers.

The application of MEM and MUSIC is aimed at helping validate the Capon processing. A description of MEM and its performance compared to the Capon method and the MUSIC algorithm via simple numerical simulations is shown in the Appendix. These simulations demonstrate that the performance of the two parametric methods is better than the performance of the Capon method for the detection of layers which are very thin when compared to the initial rangeresolution used. In particular, MEM provides a better power of separation and is less sensitive to SNR than the Capon 

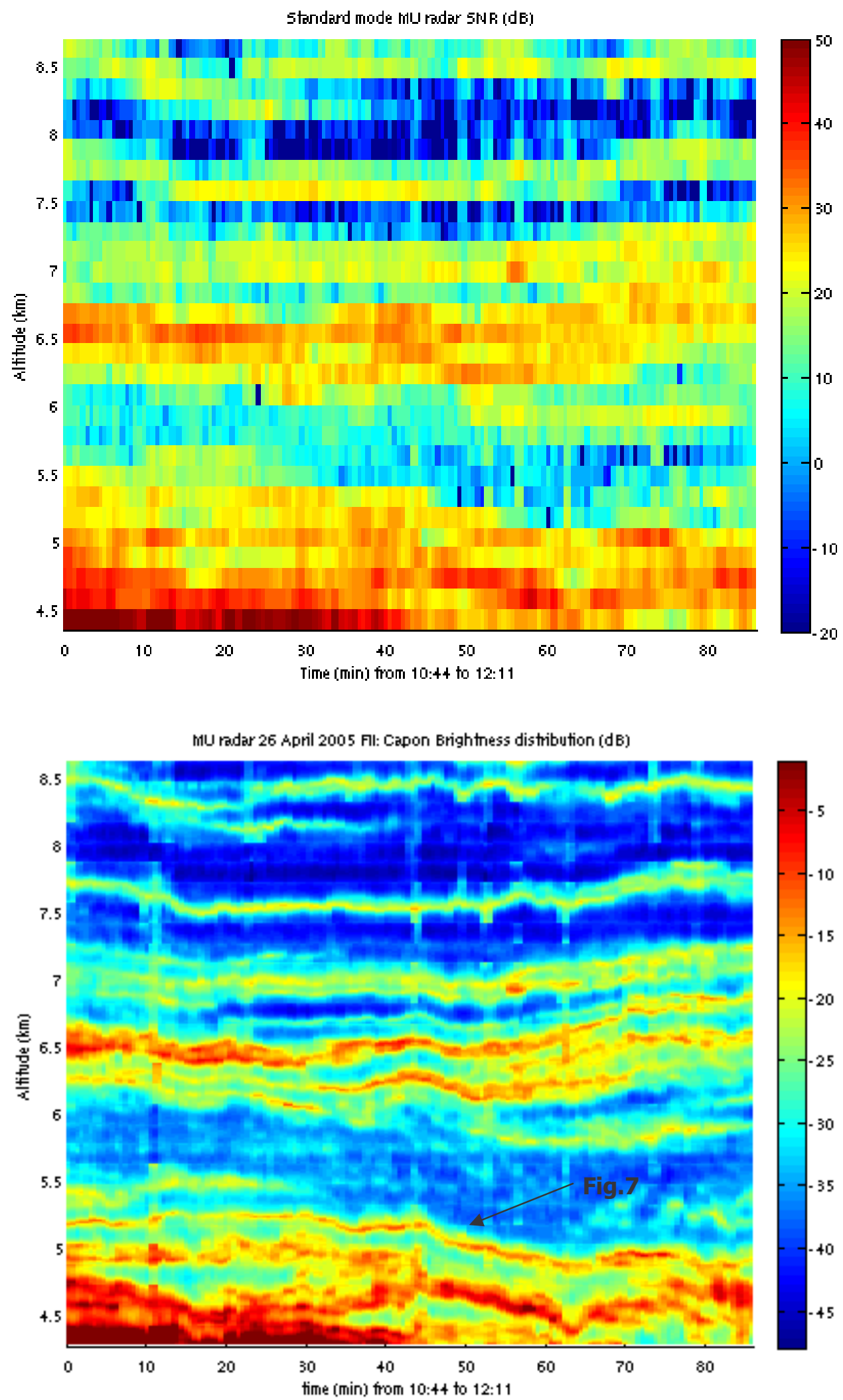

Fig. 1. (Top) Observations in standard mode (4.35-8.7 km). (Bottom) Observations after Capon processing. 
method. However, in the case of thick layers, MEM and the MUSIC algorithm always produce false sharp peaks of brightness which will be wrongly interpreted as very thin layers. Even though the distribution of the peaks gives some information on the vertical extent of the real thick layer, it cannot be interpreted without ambiguity. Such a problem of interpretation does not occur with the Capon method since this method is not constrained by the hypotheses of narrow sources. Hence, the parametric methods should be understood as complementary and should not be considered as alternatives to the Capon method. They can help in the interpretation of the Capon images when both parametric and non-parametric methods provide similar patterns.

\subsection{Experimental configuration and data processing}

The experiment was performed on 26 April 2005, 10:4412:11 LT. Five equally-spaced frequencies from 46.0 to $47.0 \mathrm{MHz}$, i.e. $\Delta f=0.25 \mathrm{MHz}$, were selected. A single vertically-directed transmit beam (beamwidth $3.6^{\circ}$ ) was used and range sampling was performed from $4.35 \mathrm{~km}$ to $23.4 \mathrm{~km}$ with a step of $150 \mathrm{~m}$. Coherent integration and time resolution were 256 and $0.51 \mathrm{~s}$, respectively. The data were collected without gaps between records and with an SNR as high as possible for optimal application of the processing methods. For this purpose, only a vertically-directed beam was used, to the detriment of the estimation of other parameters (e.g. the horizontal wind, which can be obtained by swinging the radar beam in at least 3 directions). Owing to this configuration, observations could be obtained up to about $22.20 \mathrm{~km}$ (no echoes were observed between 22.20 and $23.4 \mathrm{~km}$ ).

Almost $5 \times 5$ unnormalized covariance matrices were estimated using a time series of 128 points (corresponding here to about $1 \mathrm{~min} 5 \mathrm{~s}$ ) every 64 points for better continuity between estimates. SNR was estimated using the moment method and noise estimates from the furthest radar gate, where the signal is found to be weak. When corrupted by airplane echoes, the segments of the time series have been replaced by a linear interpolation between points assumed not to be affected. Capon and MEM patterns have been calculated using Eqs. (A3) and (A2), respectively, and the position of the peaks provided by the MUSIC algorithm has been deduced from the MUSIC patterns given by Eq. (A5), arbitrarily assuming 2 sources. The edge effects resulting from the radar range-weighting function $W^{2}(z)$ have been corrected, as described in Luce et al. (2001a), by dividing the obtained patterns by $W^{2}(z)=\exp \left(-z^{2} / \sigma_{z}^{2}\right)$, where $\sigma_{z}=\Delta r / 2=75 \mathrm{~m}$. The range-scanning for the Capon, MEM and MUSIC methods was performed independently in each $150-\mathrm{m}$ range gate every $1 \mathrm{~m}$. This step does not imply that estimates separated by 1 meter are independent. Rather it insures that no peak has been missed or no position has been significantly biased, especially for the MUSIC algorithm which can produce extremely sharp peaks. Finally, the dual-FDI method, using $\Delta f=1 \mathrm{MHz}$, has been applied (using Franke's (1990) formulas), in order to emphasize the improvements obtained with FII, and the same plotting representation has been used for easier comparisons with FII patterns.

\section{Observational results}

\subsection{Capon imaging in the troposphere and stratosphere}

For an easy analysis, the height-time plots of the images are presented in Figs. 1 to 4, showing results for $4.35-8.7 \mathrm{~km}$, $8.7-13.20 \mathrm{~km}, 13.2-17.70 \mathrm{~km}$ and $17.7-22.20 \mathrm{~km}$, respectively, including the patterns obtained with the standard processing at a resolution of $150 \mathrm{~m}$ (top) and the corresponding patterns after Capon processing (bottom). According to the radio soundings performed by meteorological stations around the MU radar site (not shown), the tropopause altitude was around $11-12 \mathrm{~km}$. The strong echoing layer detected between 11 and $11.5 \mathrm{~km}$ by the MU radar and the thick altitude range $(\sim 1.5-2.0 \mathrm{~km})$ of weak echoes (Fig. 2$)$ may thus be a signature of the tropopause altitude, usually associated with a sharp background temperature increase, and of the upper troposphere, usually associated with very low static stability, respectively.

Some artifacts can be noted, for example, around 10 and 63 min in Fig. 1, due to residual airplane echoes that still persist despite the preliminary rejection treatment. Unrealistic peaks are also noticeable at the edges of the theoretical range gate (i.e. every $150 \mathrm{~m}$ ) when the pattern is near its minimum (blue color). They result from the edge-effect correction that should not be applied when the noise background is reached since it is not affected by the radar range-weighting function. The correction should only be applied when there actually is a layer near the edge of the range gate, but this information is not known in advance. Therefore, these edge effects should not be considered when analyzing the results.

For the first time, significant patterns in interferometric observational mode have been obtained up to about $22 \mathrm{~km}$, i.e., both in the troposphere and lower stratosphere. The effectiveness of the FII processing is very clear from Figs. 1 to 4 when comparing the Capon pattern with the standard single-frequency mode pattern. The Capon pattern reveals individual and persistent echoing layers, sometimes much thinner than the range resolution used, which are often difficult and sometimes impossible to resolve in standard mode. It thus confirms the strong layering of the lower atmosphere, and especially of the lower stratosphere at vertical scales of $10^{1} \sim 10^{2} \mathrm{~m}$. This property was expected but this is the first time that it has been directly observed by a VHF ST radar in the lower stratosphere at such a high vertical resolution. It is then worthwhile to note that the possible apparent intermittency of the echoes observed with the standard mode at a range resolution of $150 \mathrm{~m}$ results from the lack of range resolution. Some striking examples: 

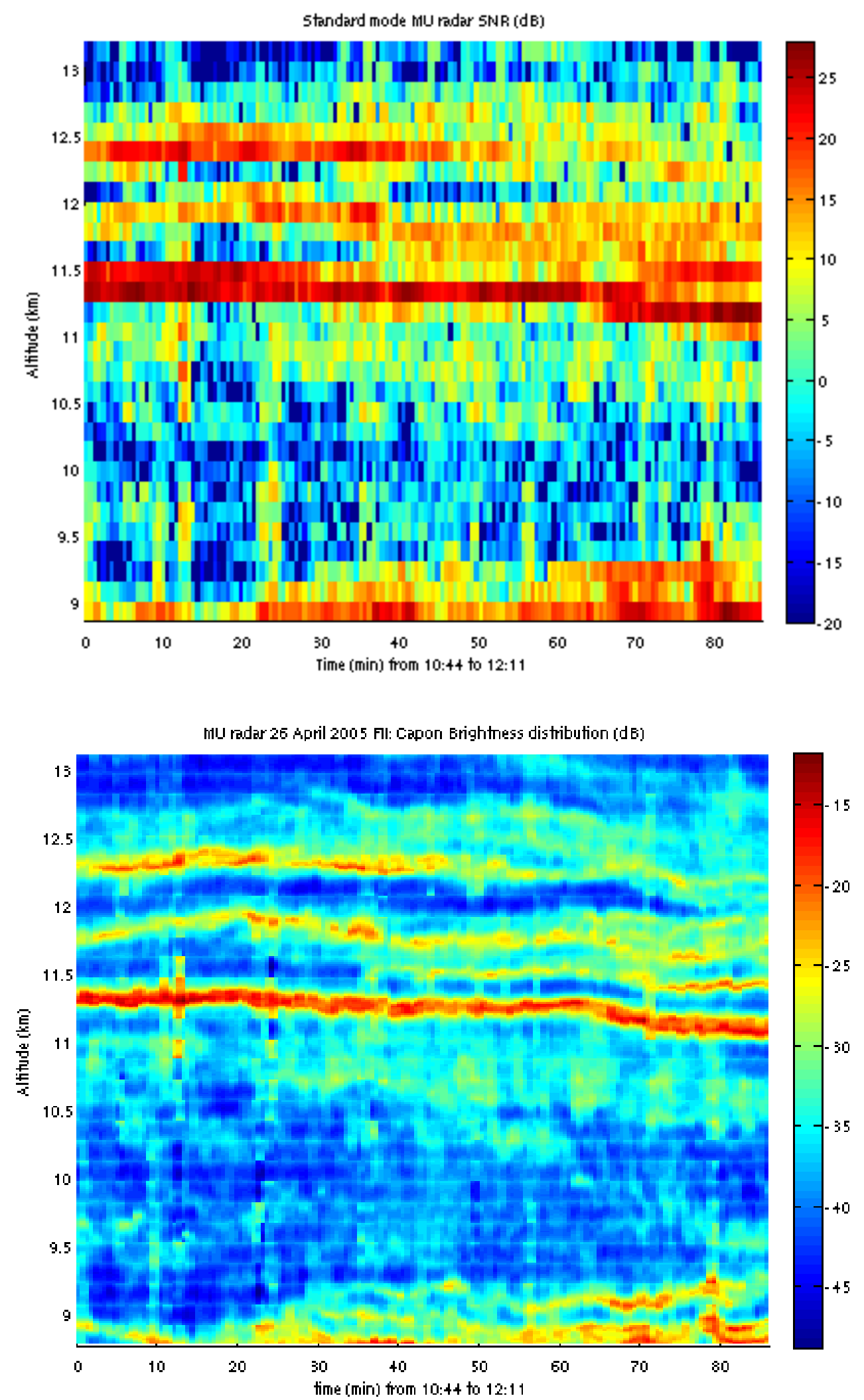

Fig. 2. Same as Fig. 1 for 8.7 to $13.05 \mathrm{~km}$. 

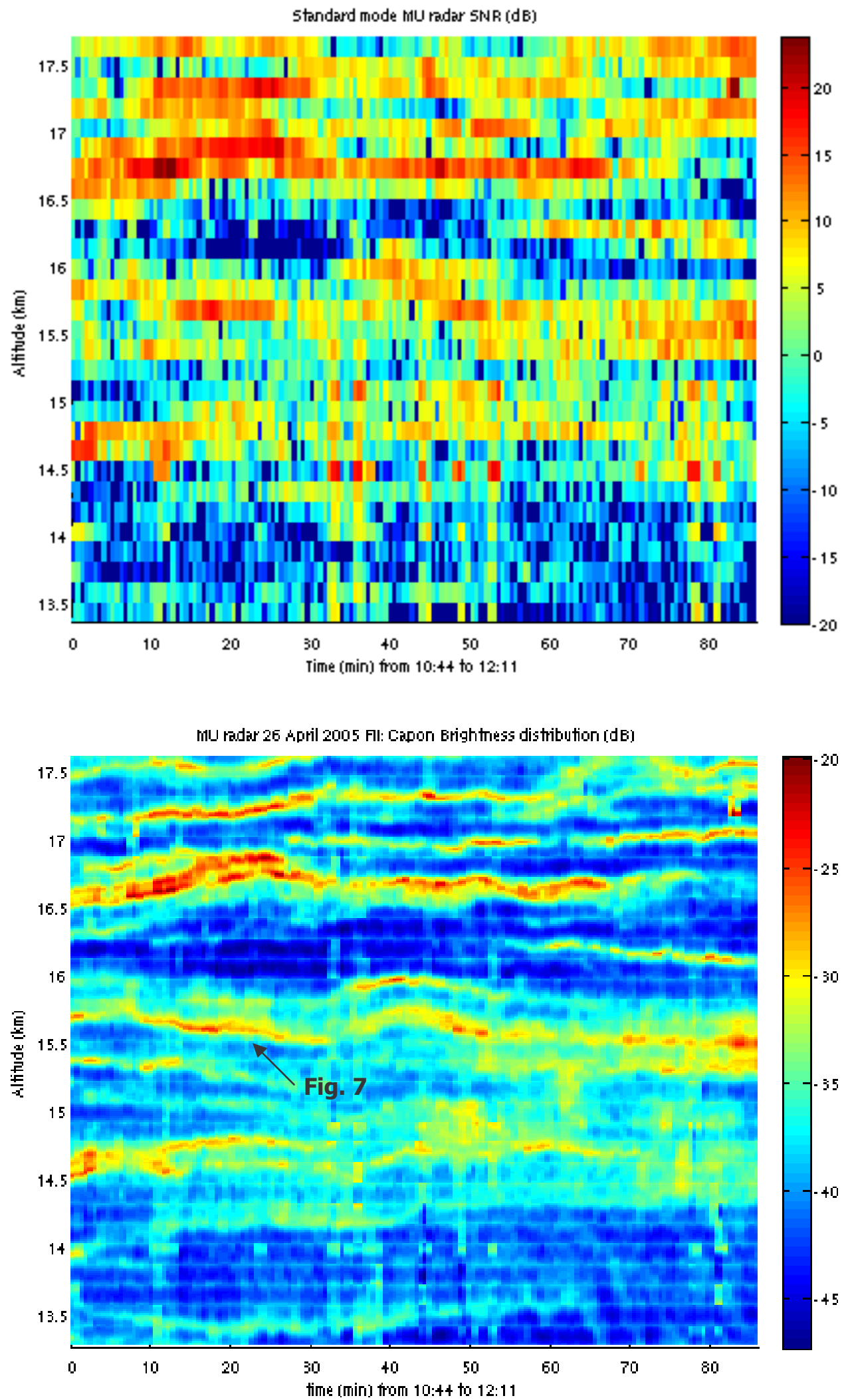

Fig. 3. Same as Fig. 1 for 13.05 to $17.55 \mathrm{~km}$. 

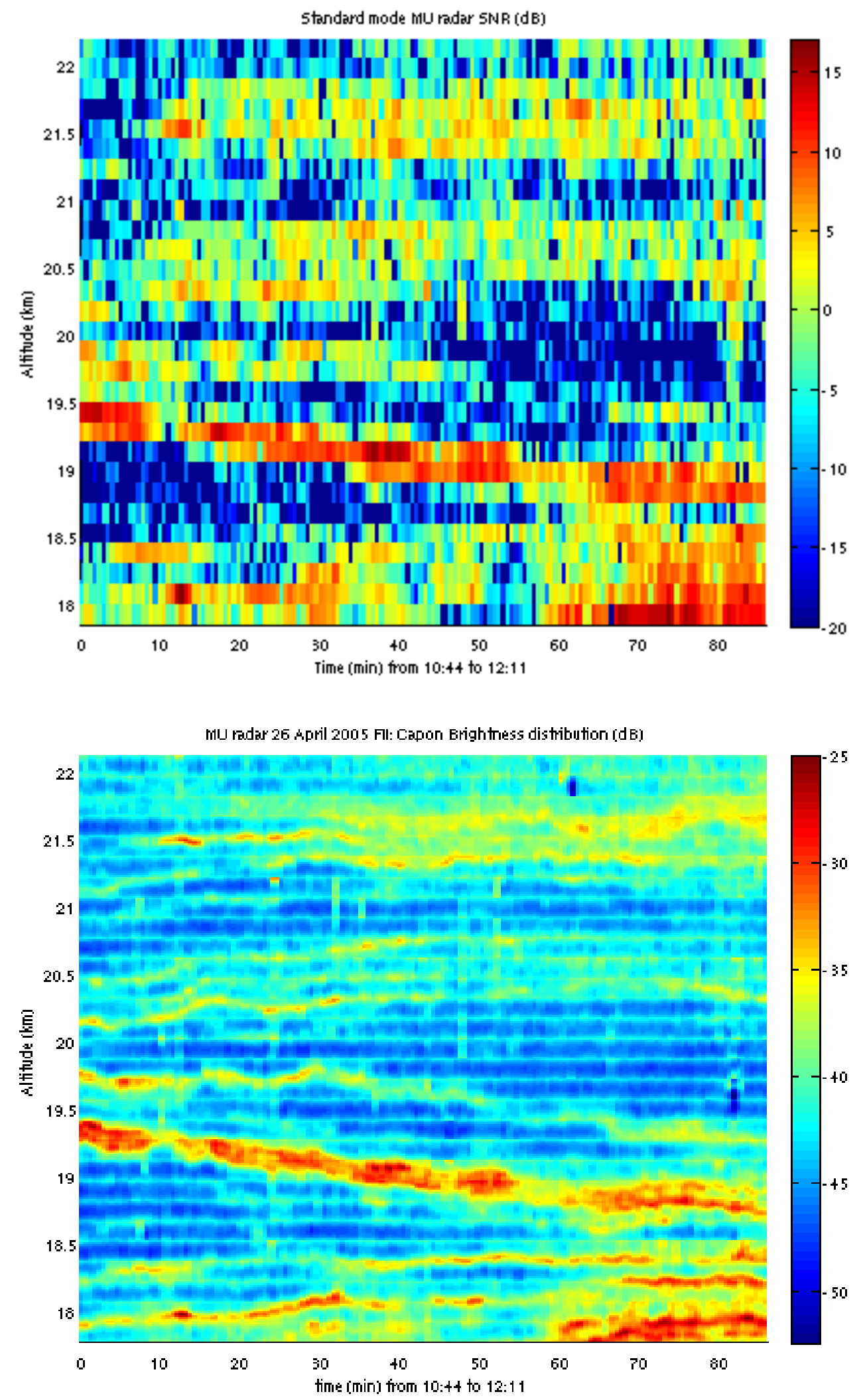

Fig. 4. Same as Fig. 1 for 17.55 to $22.05 \mathrm{~km}$. 
- In the troposphere, layered structures are revealed by the Capon pattern below $5.5 \mathrm{~km}$ and between $6.2 \mathrm{~km}$ and $7 \mathrm{~km}$, while the standard pattern rather provides quite thick and intermittent structures (Fig. 1). These layered structures are plausible and the Capon pattern radically changes our interpretation of the radar echoes measured in standard mode in these altitude ranges. The curved parts of the two maxima between $30 \mathrm{~min}$ and $50 \mathrm{~min}$ and around $6.3 \mathrm{~km}$ are clearly separated by a deep minimum that cannot be distinguished in standard mode. The latter merely shows a short-lived thick maximum, very likely due to smoothing effects by the radar rangeweighting function. The intense echoes below $5.5 \mathrm{~km}$ are in fact overhung by a thin layer that quickly moves downwards from $45 \mathrm{~min}$. It can only be roughly approximated from the standard mode observations.

- Above the tropopause, layers in the Capon pattern separated by about $500 \mathrm{~m}$ can be clearly distinguished around $11.7 \mathrm{~km}$ and $12.3 \mathrm{~km}$ at the beginning of the experiment (Fig. 2). Impossible to identify in the standard pattern, the Capon pattern also shows the appearance of a layer at $38 \mathrm{~min}$ and at mid-distance between the tropopause layer and the layer identified at $11.7 \mathrm{~km}$. Also, the standard mode seems to indicate that the tropopause layer is divided into two around $70 \mathrm{~min}$, but the Capon pattern clearly indicates that there is no relation between the two structures. Above $20-\mathrm{km}$ altitude, three consecutive layers, separated by less than $100 \mathrm{~m}$ and oscillating together are resolved in the Capon pattern (Fig. 4). This feature is compatible with the observations of strong temperature gradient sheets observed in groups of high-resolution, in-situ temperature profiles (e.g. Dalaudier et al., 1994, and mainly in the MUTSI temperature profiles, Fig. 4 of Gavrilov et al., 2005).

Some layers in the Capon pattern appear sometimes quite thick (of the order of the range resolution used, i.e. about $150 \mathrm{~m}$ ). This is the case for the tropopause layer around $11.3 \mathrm{~km}$. Another thick layer of variable intensity and thickness apparently moves downward between $19 \mathrm{~km}$ and $19.5 \mathrm{~km}$ and internal structures that look like turbulent billows can be seen, especially after $60 \mathrm{~min}$. The lack of complementary information prevents us from proving their existence. However, considering the performance of the technique, it is reasonable to suggest that this interpretation is plausible. Also, analyses of the raw spectral width of the Doppler spectra clearly indicate a strong enlargement in the altitude range of this thick layer (not shown) while no clear signature of width enhancement is found for the thinner layers seen above. This observation may support the turbulent nature of the layer around $19 \mathrm{~km}$.

\subsection{Comparisons of processing methods}

For comparison of the Capon pattern with MEM, MUSIC and dual-FDI patterns, we selected a close-up between $5.8 \mathrm{~km}$ and $7.3 \mathrm{~km}$. The results are shown in Fig. 5. The top panel shows the Capon pattern, and the dots indicate the position of all the brightness peaks. Horizontal solid lines indicate the limits of the theoretical range gates. The center panel of Fig. 5 shows the MEM pattern, and the dots show the position of the maxima provided by the MUSIC algorithm, assuming 2 sources. The choice of 2 sources is arbitrary but is selected because it corresponds to the number of peaks sometimes resolved by the Capon method and MEM. The bottom panel of Fig. 5 shows the results of dual-FDI processing, using the same representation as for the FII processing. The dots indicate the peak positions provided by dual-FDI.

\subsubsection{Comparison with MEM and the MUSIC algorithm}

The Capon pattern often reveals a single peak in one range gate. For example, a very thin layer is found mainly in gate 7 and appears around $20 \mathrm{~min}$. But sometimes, it reveals two maxima in one range gate (e.g. in gate 5 between $10 \mathrm{~min}$ and $30 \mathrm{~min}$, and gates 3 and 8 between $50 \mathrm{~min}$ and $60 \mathrm{~min}$ ):

- Around $7 \mathrm{~km}$, a double echoing layer is observed during the entire observation period. The distance between the two maxima is typically $75-150 \mathrm{~m}$. Interestingly, the two maxima are alternately found in two consecutive gates (i.e. one maximum is found in gates 8 and 9) and within a single gate (two maxima are found in gate 8 , around $10 \mathrm{~min}, 30 \mathrm{~min}$ and $50 \mathrm{~min}$ ), giving extra credence to the reality of the double echoing layer, i.e. the structures are not artifacts of the processing method.

- Another interesting feature is the curved structure around $6.2 \mathrm{~km}$. It appears around $6 \mathrm{~min}$ and both its thickness and intensity increase with time, until it definitively becomes a double structure from about $50 \mathrm{~min}$ and then slowly disappears.

Comparing with the results of the other methods, it clearly appears that the MEM pattern is extremely similar to the Capon pattern. The MEM pattern, however, appears much more fluctuating, compatible with the properties of the method regardless of the layer thickness (see Appendix). The curved structure around $6.2 \mathrm{~km}$ reveals a double structure earlier (around $38 \mathrm{~min}$ ) than the Capon pattern. A sample of the Capon brightness distribution for 5 successive records is given in Fig. 6, as well as the positions given by MEM and the MUSIC algorithm. The positions agree quite well and the distance between the two peaks is typically between $60 \mathrm{~m}$ and $90 \mathrm{~m}$ for the three methods. This result gives extra credence to the existence of a distribution more complex than a single layer (likely a double layer) in the radar gate. Even though the MEM and MUSIC patterns cannot be interpreted without ambiguity when several peaks are detected, 

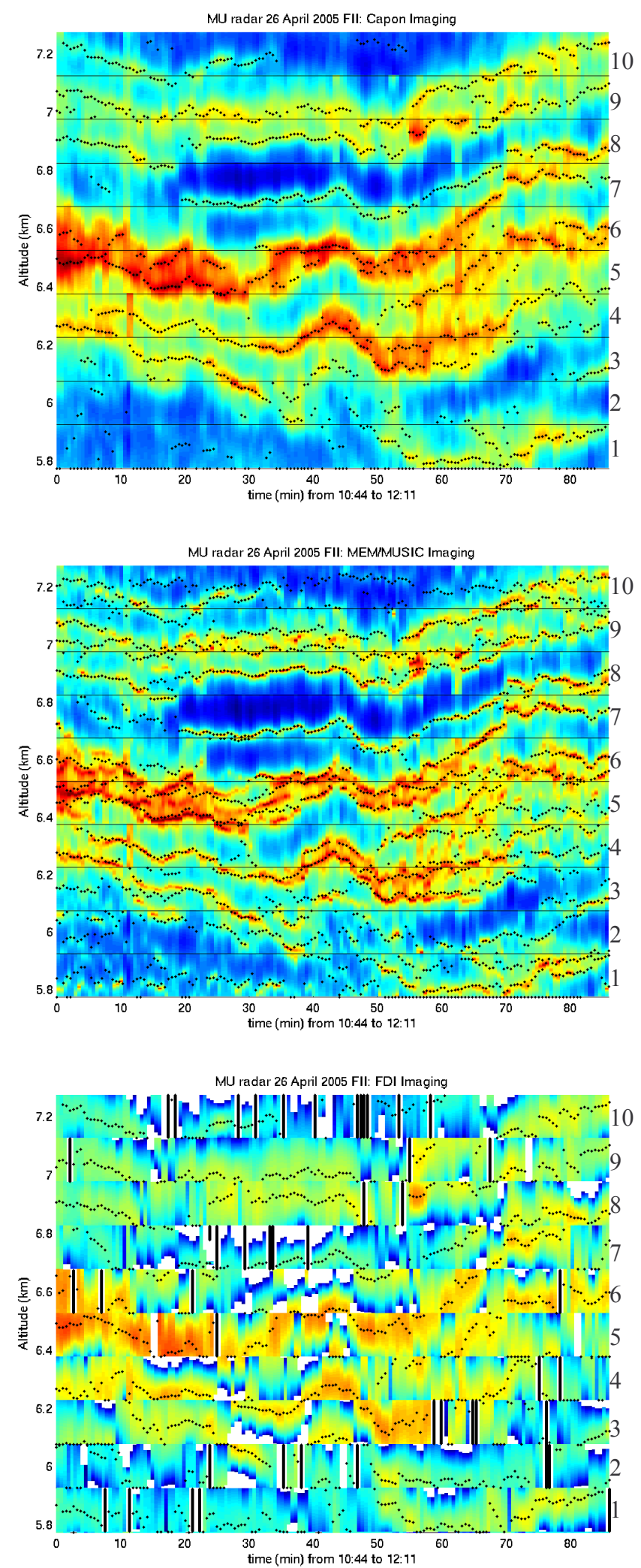

Fig. 5. Close-up of high-resolution patterns in the troposphere obtained from (a) the Capon method, (b) MEM, while dots indicate the positions of the peaks obtained with the MUSIC algorithm, (c) the dual-FDI. 


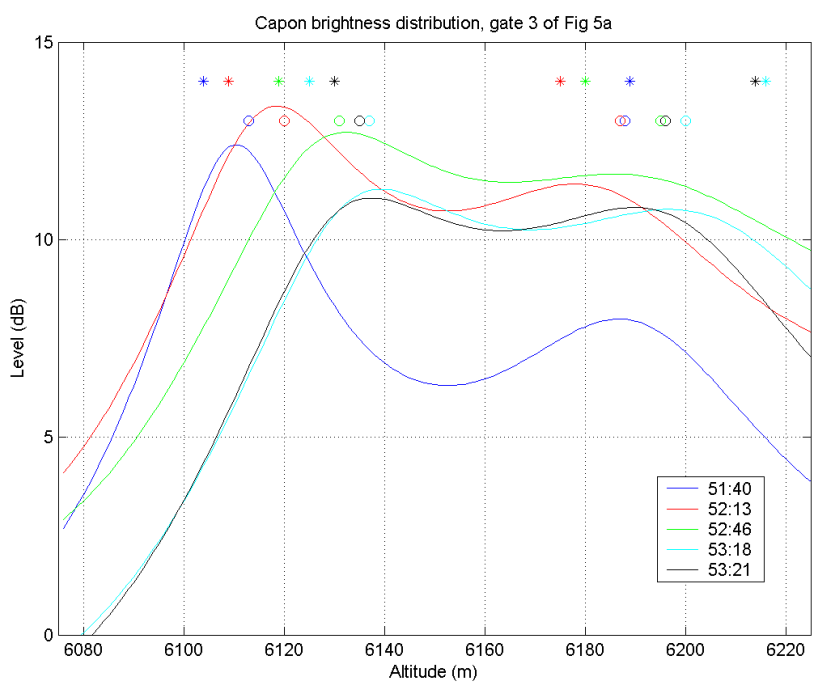

Fig. 6. Capon brightness distribution for 5 consecutive records from time 51:40 to 53:21 and for gate 3 of Fig. 5a where a double peak is observed. The stars and circles indicate the corresponding positions given by MEM and the MUSIC algorithm, respectively.

the evolution of the structure may suggest that the resolution performance of the Capon processing were not sufficient for separating the two sources before about $45 \mathrm{~min}$. It is speculated that the radar may have detected the evolution of a turbulent layer growing with time. Because of the mixing effects, the radar would mainly detect, after a while, the edges of the mixing layer where the refractive index irregularities are enhanced. If this plausible interpretation is correct, it would be the first time that such an event at vertical scale, significantly smaller than $100 \mathrm{~m}$, would have been directly observed by a VHF radar.

The MUSIC algorithm also provides almost exactly the same positions as MEM and Capon processing. In particular, the three methods give almost the same single layer pattern in gate 7 from $20 \mathrm{~min}$. Only one source is found by MUSIC while 2 sources have been assumed. This phenomenon can occur when the atmospheric layer is really thin and unique, as the dark blue color above indicates, the two sources being too close to one another to be separated in the range scanning. A similar event can be found in gate 8 at the same time. For these 2 examples, the strong similarities between the Capon pattern and the patterns of the parametric methods highly suggest that the methods reveal a very thin atmospheric structure without ambiguity.

\subsubsection{Comparison with dual-FDI}

The dual-FDI pattern seen in the bottom panel of Fig. 5 shows roughly similar structures, but the time and spatial continuity is very poor compared to pattern obtained from the FII processing. FDI processing also produces "ghost" layers resulting from the contribution of atmospheric scatter- ers outside the theoretical range gate and the $2 \pi$ phase periodicity of the FDI processing (e.g. gate 3 between 0 min and $10 \mathrm{~min}$, gates 3 to 7 between $40 \mathrm{~min}$ and $50 \mathrm{~min}$ ). However, it is interesting to note that the dual-FDI pattern confirms the double structure in gates 8 and 9 until about $45 \mathrm{~min}$. Because the vertical separation between the two structures varies with time, they really correspond to distinct atmospheric structures. By definition, dual-FDI can only detect single layers, and necessarily fails when several layers are embedded within the range gate. The failure can manifest itself as a non-meaningful layer thickness, but can also fail entirely to provide a value for the thickness, as demonstrated by Luce et al. (1999). The presence of a couple of layers can produce a measured signal coherence smaller than it should be from the theory for a single layer. Then the FDI algorithm has no solution. This occurs in gate 3 between $60 \mathrm{~min}$ and $70 \mathrm{~min}$ (the absence of a solution is indicated by a white rectangle) and around $50 \mathrm{~min}$ in gate 8 where a double structure is found by the FII processing. (Other failures can also occur when the corrected FDI coherence from SNR exceeds unity, in regions of weak SNR.)

\subsubsection{Layer thickness estimated from the Capon method}

Time evolution of the position and thickness of the layers estimated from the Capon method, the associated peak of brightness and the signal-to-noise ratio (SNR) observed in the corresponding range gate is given in Fig. 7 for two selected layers in the troposphere and stratosphere. For easy comparison, the thickness has been calculated by fitting a Gaussian curve to each maximum of the Capon pattern. The number of points used for the fitting is 10 , arbitrarily around the maximum. It was verified that the choice of the number of points does not qualitatively affect the results. The thickness has been defined as twice the standard deviation of the Gaussian curve, the same definition as for the dual-FDI thickness. Chilson et al. (2003) performed a similar analysis with UHF data but used twice the square root of the second central moment for estimating the layer thickness. The absence of points usually corresponds to the absence of a peak in the range gate or to a non-realistic peak (bad continuity in time). The horizontal blue dotted-dashed line corresponds to a SNR threshold above which SNR effects on the estimation of the layer thickness are expected to be weak (see Appendix). This threshold has been taken arbitrarily as $10 \mathrm{~dB}$. For the tropospheric layer (around $5.13 \mathrm{~km}$ ), the associated SNR is roughly larger than $20 \mathrm{~dB}$, while it is about $10 \mathrm{~dB}$ or sometimes less for the stratospheric layer (around $15.59 \mathrm{~km}$ ).

The selected tropospheric layer has sometimes a thickness as small as about $20 \mathrm{~m}$. It appears to deepen significantly around 10, 16, 20 and $45 \mathrm{~min}$. These layer increases are related to SNR minima for the three first cases, of about $20 \mathrm{~dB}$. However, at such high SNR, blurring effects are not expected to be strong, according to the simulations shown in the Appendix. Thus, it is believed that the thickness variations are 


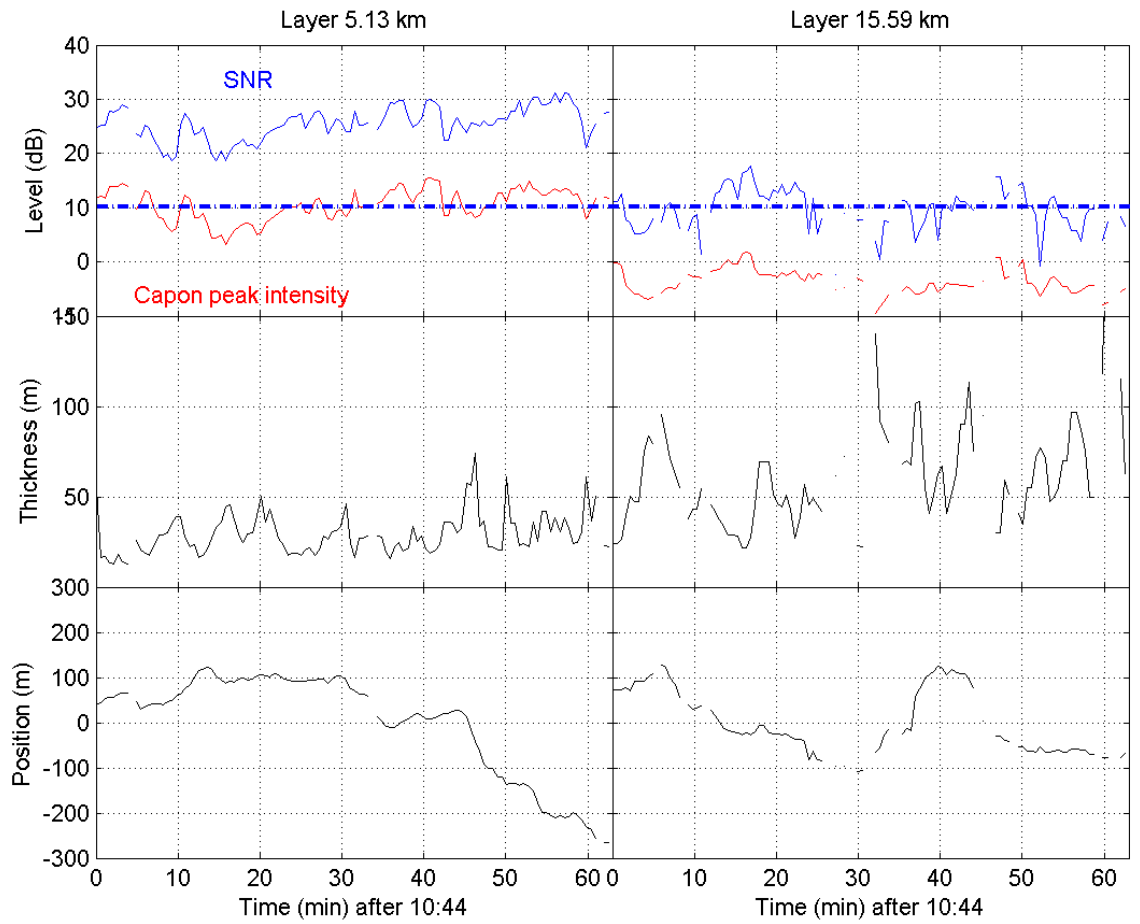

Fig. 7. Time evolution of the position (bottom), thickness (center) and maximum peak intensity and SNR in dB (top) for 2 single layers detected by the Capon method at mean altitudes of $5.13 \mathrm{~km}$ and $15.59 \mathrm{~km}$. The layers are not clearly defined after around $63 \mathrm{~min}$ for both cases.
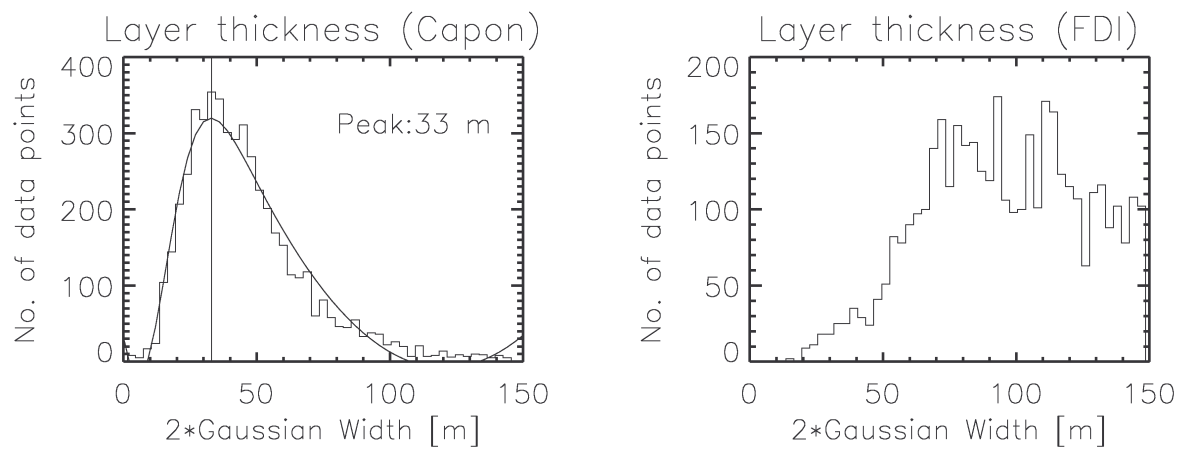

Fig. 8. Comparison between the histograms of thickness distributions estimated from the Capon method (left) and the FDI algorithm (right) for all the available data set.

rather due to atmospheric effects. The increase in thickness around $45 \mathrm{~min}$ is associated with a small minimum in peak brightness but not in SNR (larger than $20 \mathrm{~dB}$ ), indicating that this thickness increase is rather due to an effect of the fast downward motion of the layer. Thus, the thickness of the layer is likely a combination of its real thickness and of its vertical displacement during the data acquisition time.

The selected stratospheric layer appears much thicker than the tropospheric layer. The low-SNR effects on the layer thickness are much more evident for this case. The SNR minima (below $10 \mathrm{~dB}$ ) indeed clearly correspond to large in- creases of thickness. Thus, it is expected that blurring effects are dominant at stratospheric altitude ranges where SNR is usually smaller than $15 \mathrm{~dB}$ at the altitudes of the resolved layers. Thus, it is possible that the stratospheric layers are even thinner than the thickness suggested by Figs. 3 and 4 .

Figure 8 shows histograms of layer thickness estimates from the Capon method and FDI algorithm for the total data set. Their peaks are at $33-36 \mathrm{~m}$ and $70-80 \mathrm{~m}$, respectively. It is clear that the thickness in the Capon patterns is more compatible with the typical thickness of the temperature and humidity gradient sheets observed by high-resolution, in-situ 
techniques (Dalaudier et al., 1994; Muschinski and Wode, 1998). The thickness estimated from the FDI algorithm only denotes a characteristic already emphasized by Luce et al. (2001c): the average is close to half of the radar rangeresolution and is thus merely an artifact of the method.

In Fig. 8, the layer thickness histogram was calculated from 40 records with 256 points, using 2 times oversampling to smooth the results. The average thickness was $47 \mathrm{~m}$. Processing of the data as 64-point or 128-point records led to histogram peaks at $24 \mathrm{~m}$ and $33 \mathrm{~m}$, and average thicknesses of $41 \mathrm{~m}$ and $45 \mathrm{~m}$, respectively. The estimated thickness thus shows a slight dependence on the time over which data processing is carried out, as expected, owing to both statistical variation and vertical movement of the layers due to various dynamic effects, such as gravity waves, convection or fronts.

\section{Conclusions}

Range imaging of the lower atmosphere using the FII technique was performed for the first time up to $\sim 22 \mathrm{~km}$ in the lower stratosphere with the new 46.5-MHz Middle and Upper atmosphere (MU) radar system at Shigaraki, Japan, in April 2005. The Capon patterns revealed persistent structures much thinner than $150 \mathrm{~m}$, very often corroborated by the parametric MEM and MUSIC algorithm, only very roughly suggested by the classical FDI technique, and impossible to resolve with the standard 150 -m mode. This work thus gives credence into the effectiveness of the FII technique. The Capon images confirm the strong layering of the lower stratosphere at vertical scales of $10^{1} \sim 10^{2} \mathrm{~m}$, i.e. at the highest vertical resolution that could be achieved with a VHF radar.

Such a technique of more reliably estimating layer thickness is useful in two important ways. Firstly, radar observations can more easily be reconciled with those of other instruments, allowing radar observations to be used operationally for observing thin-layer structures, and secondly, continuous observations of these structures and the better resolving of their changes in time would permit us to ascertain local small-scale dynamics of the tropo-stratosphere, and determine the generation mechanisms of the layers with greater accuracy.

High-resolution imaging with the new MU radar imaging system will permit a better analysis of the temperature gradient sheets and thin turbulent layers by VHF ST radars. Consequently, future studies will be carried out on interpreting the structures resolved by the FII mode.

\section{Appendix A Maximum Entropy Method}

The Fourier-based imaging method proposed by Palmer et al. (1999) and Luce et al. (2001) is based on the use of the FFT to estimate the brightness distribution. In terms of Z-transforms, the model brightness distribution can only have zeros in the Z-plane. This refers to the moving average (MA), all-zero model. A different estimation can be provided by an auto-regressive (AR), all-poles model, also called Maximum Entropy Method (e.g. Candy, 1988). The estimated brightness distribution can have the form (in terms of Z-transforms):

$\mathrm{B}_{\mathrm{AR}}(\mathrm{Z}) \propto \frac{1}{\left|1+\sum_{\mathrm{k}=1}^{\mathrm{N}} \mathrm{a}_{\mathrm{k}} \mathrm{Z}^{\mathrm{k}}\right|^{2}}$.

Since this estimate can have poles, it is well-adapted to brightness distributions with sharp peaks. For application to range imaging, the MEM brightness distribution can be written as (Nickel, 1988; after only slight modifications of applications to antenna array processing):

$\mathrm{B}_{\mathrm{MEM}}(\mathrm{z})=\mathbf{e}_{1}^{*} \mathbf{R}^{-1} \mathbf{e}_{1} /\left|\mathbf{e}_{1}^{*} \mathbf{R}^{-1} \mathbf{a}(\mathrm{z})\right|^{2}$,

where $\mathbf{a}(z)=\left[\begin{array}{lll}1 & \exp (j \Delta k z) & \exp (j 2 \Delta k z) \ldots \exp (j(N-\end{array}\right.$ 1) $\Delta k z)]^{T}$ is the scanning vector of dimension $\mathrm{N}$ at position $\mathrm{z}$, $\mathbf{R}$ is the $\mathrm{N} \times \mathrm{N}$ covariance matrix of the received signals at $\mathrm{N}$ different frequencies, $\mathbf{e}_{1}=(1,0, \ldots 0)^{\mathrm{T}}$ is a unit vector, and $*$ represents the conjugate transpose operator. When Eq. (A2) is given in terms of Z-transforms, an expression of the form of Eq. (A1) is obtained. As reported by Nickel (1988), the Capon pattern given by, for example, Palmer et al. (1999) and Luce et al. (2001a) is:

$\mathrm{B}_{\mathrm{cap}}(\mathrm{z})=\left(\mathbf{a}(\mathrm{z}) \mathbf{R}^{-1} \mathbf{a}^{*}(\mathrm{z})\right)^{-1}$,

which corresponds to a harmonic averaging of the MEM pattern such that

$\mathrm{B}_{\text {cap }}(\mathrm{z})=\frac{1}{\mathrm{~N}} \sum_{\mathrm{k}=1}^{\mathrm{N}} \mathrm{B}_{\mathrm{MEM}, \mathrm{k}}^{-1}(\mathrm{z})$,

where $\mathrm{B}_{\text {MEM,k }}$ is the MEM pattern obtained from the last $\mathrm{k}$ frequencies (see also Stoïca and Moses, 202-204, 1997). Thus, the MEM pattern should be affected more by statistical errors (i.e. the MEM pattern should show greater fluctuations) but the resolution performance should be better. The MUSIC algorithm consists first in estimating the eigenvectors of the covariance matrix. For $\mathrm{M}<\mathrm{N}$ discrete sources, $\mathrm{M}$ sources are associated with $\mathrm{M}$ eigenvectors (corresponding to the Signal subspace). The last N-M eigenvectors are related to the Noise subspace. If we note $\mathbf{T}=\left[\begin{array}{ll}\mathbf{t}_{1} & \mathbf{t}_{2} \ldots \mathbf{t}_{N-M}\end{array}\right]$ the $\mathrm{N} \times(\mathrm{N}-\mathrm{M})$ matrix of Noise eigenvectors, then the MUSIC pattern is given by:

$\operatorname{B}_{\text {MUSIC }}(\mathrm{z})=\left(\mathbf{a}^{*}(\mathrm{z}) \mathbf{T T}^{*} \mathbf{a}(\mathrm{z})\right)^{-1}$.

In order to assess the performance of MEM with respect to the Capon method and the MUSIC algorithm, simple numerical simulations have been performed. The modeling scheme used for these simulations has already been presented by 

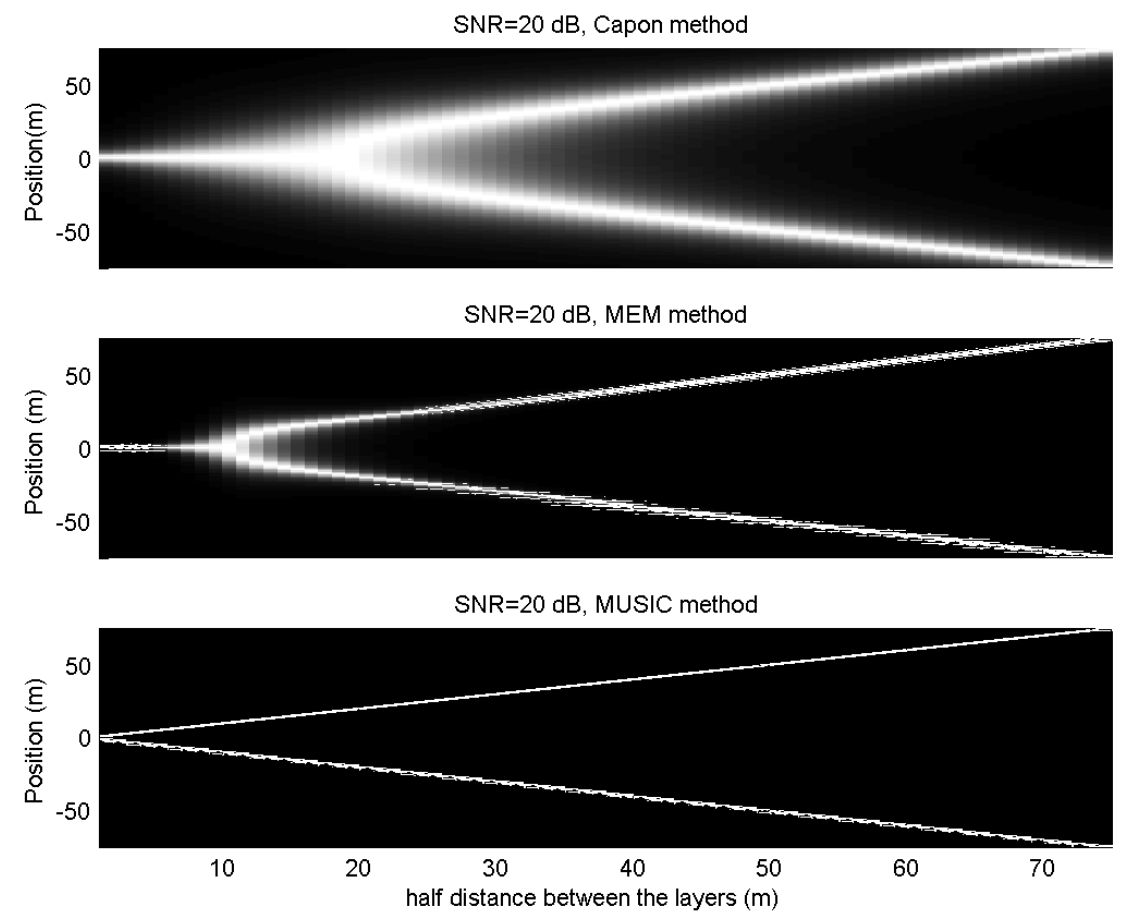

Fig. A1. Results of simulations for the Capon method (top), MEM (center) and MUSIC algorithm (bottom), assuming SNR $=20 \mathrm{~dB}$ and two "infinitely" thin layers separated by $0 \mathrm{~m}$ to $150 \mathrm{~m}$.
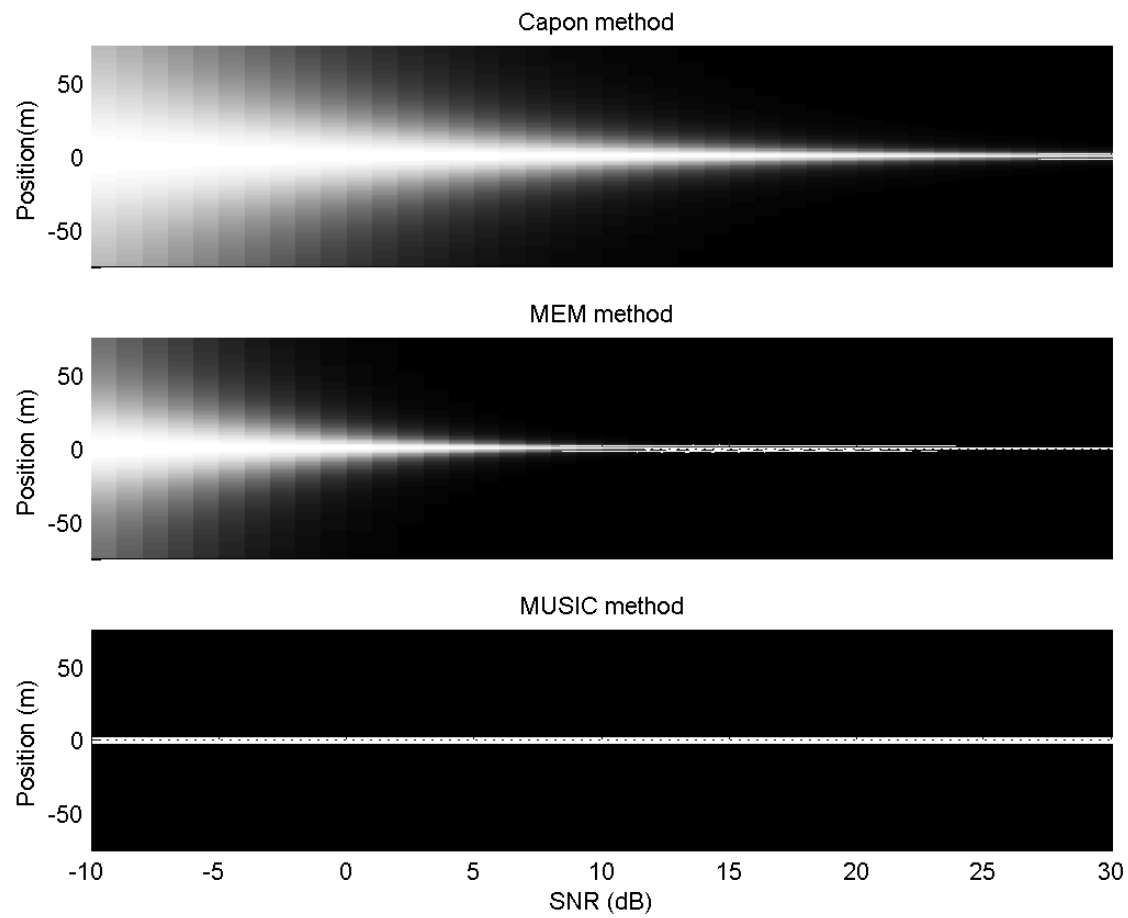

Fig. A2. Results of simulations for the Capon method (top), MEM (center) and MUSIC algorithm (bottom), assuming a single "infinitely" thin layer and for SNR from $-10 \mathrm{~dB}$ to $30 \mathrm{~dB}$. 

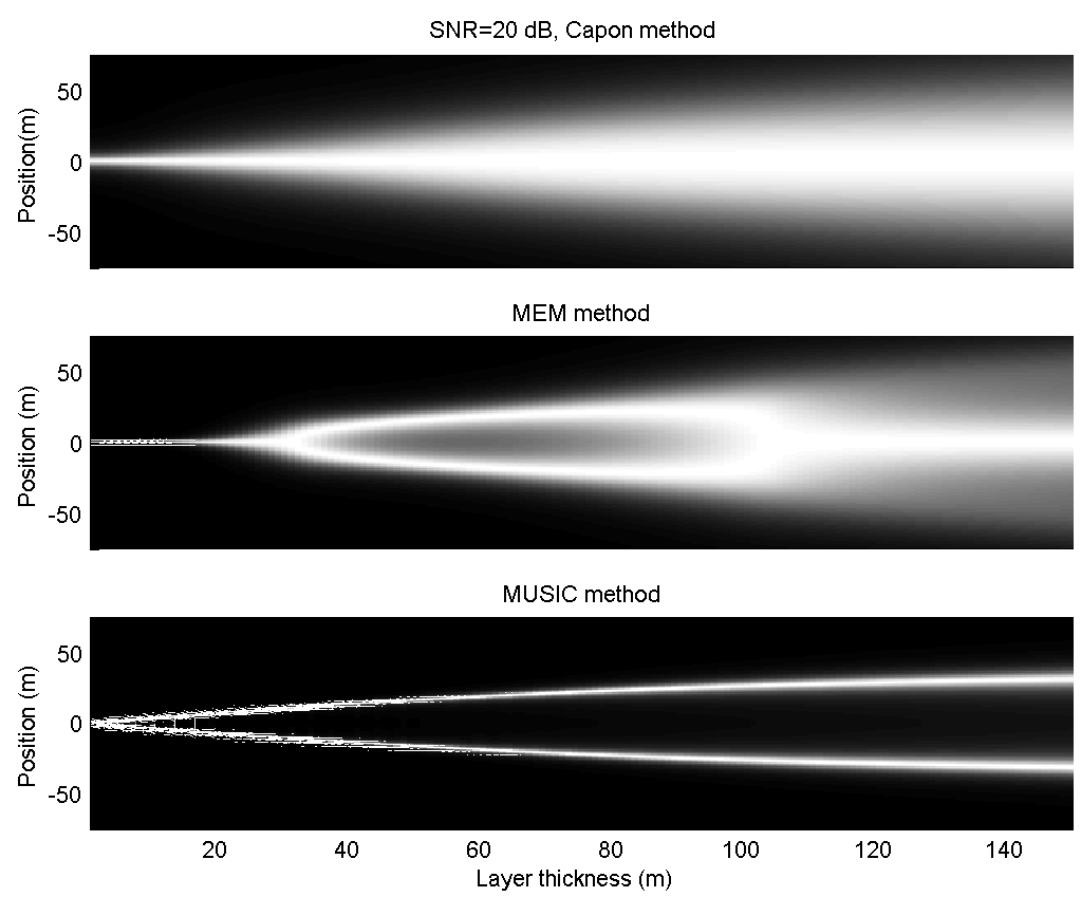

Fig. A3. Results of simulations for the Capon method (top), MEM (center) and MUSIC algorithm (bottom), assuming SNR = 20 dB and a single layer of thickness from $0 \mathrm{~m}$ to $150 \mathrm{~m}$.

Luce et al. (2001a) and Smaïni et al. (2002). It is based on the analytical calculation of the complex normalized crosscorrelation, assuming a given number of Gaussian-shaped layers embedded within the range gate (Luce et al., 1999). The thickness of the layers is defined as twice the standard deviation $\sigma$ of the Gaussian function representing the layers.

The normalized cross-correlation matrix is hence calculated for a given distribution of layers, assuming that the backscattered signals from each layer are uncorrelated. The noise effects are taken into account by assuming white noise, so that only the diagonal of the matrix is affected by an additional term $P_{N}$. The signal-to-noise ratio is thus defined as $10 \log _{10}\left(P_{S} / P_{N}\right)$, where $P_{S}=1$ is the arbitrary power, i.e. the normalized cross-correlation at 0 frequency lag. The proposed simulations show the optimal performances of the processing methods since they provide a cross-correlation matrix without estimation errors.

Contrary to Palmer et al. (1999), the effects of the wavefront curvature are not taken into account because these effects depend on the layers' height and are quite small for tropospheric altitudes and for narrow beams (e.g. Luce et al., 2000). The mean altitude of the selected layers is thus not important.

Figures A1-A3 show results of FII simulations, assuming five equally-spaced frequencies over $1 \mathrm{MHz}$ and $\Delta r=150 \mathrm{~m}$. Figure A1 shows FII patterns using the Capon method, MEM and the MUSIC algorithm (assuming 2 sources) for the case of 2 infinitely thin layers (i.e. $\sigma=0$ ) and assuming
$\mathrm{SNR}=20 \mathrm{~dB}$. As expected, MEM provides separation capabilities better than the Capon method but worse than the MUSIC algorithm. For this case, the layers are detected for a vertical separation distance of about $40 \mathrm{~m}$ with the Capon method, while only about $20 \mathrm{~m}$ is needed with MEM. Thus, in case of the detection of several very thin layers, MEM (and the MUSIC algorithm) are more adapted than the Capon method.

Figure A2 shows FII patterns using the same methods as in Fig. A1, assuming a single "infinitely thin" layer located at the center of the gate, versus SNR from $-10 \mathrm{~dB}$ to $30 \mathrm{~dB}$. The plots show that MEM is less sensitive to SNR than the Capon method. Blurring effects are noticeable from 10-20 dB with the Capon method and from $0-10 \mathrm{~dB}$ with MEM, depending on the error tolerance. For this layer model, MEM is thus more adapted than the Capon method at low SNR. One can note that the MUSIC algorithm only provides one peak despite the assumption of 2 sources.

Figure A3 shows FII patterns using the same methods as in Fig. A1, assuming SNR $=20 \mathrm{~dB}$ and a single layer of thickness from $0 \mathrm{~m}$ to $150 \mathrm{~m}$. As expected, the Capon method provides the best pattern with increasing layer thickness and detects only a single layer regardless of the thickness. Since MEM and the MUSIC algorithm are adapted to distributions with sharp peaks, they reveal "ghosts" for "thick" layers: MEM detects a double peak from a thickness of about $30 \mathrm{~m}$ onwards and then a triple peak starts to form from $100 \mathrm{~m}$ onwards. The MUSIC algorithm shows two peaks with an 
increasing separation distance when the layer thickness increases, indicating that the separation can be an indicator of the vertical extent of the thick layer. Applied on real data, MEM and the MUSIC algorithm cannot provide information on the real thickness of the structures.

In summary, each method has its advantages and drawbacks, depending on the assumed layer model. MEM shows a performance intermediate to the Capon method and the MUSIC algorithm. MEM and the MUSIC algorithm are not adapted for thick layers but can provide much better resolution performances than the Capon method when multiple thin layers are assumed. As a consequence, the application of the different methods to the same data set can provide the following information: if the Capon pattern is similar to the patterns obtained with MEM and the MUSIC algorithm (i.e. the same number of layers is found), this reinforces the hypothesis that the Capon method sees real atmospheric structures. When they differ (i.e. when MEM and MUSIC patterns show more peaks than the Capon patterns), the results can be interpreted in two different manners: either the Capon method performance is not sufficient to resolve the structures, or MEM and the MUSIC algorithm show spurious peaks.

Acknowledgements. Topical Editor F. D'Andrea thanks two referees for their help in evaluating this paper.

\section{References}

Candy, J. V.: Signal processing, The modern approach, McGrawHill, 1988.

Chilson, P. B., Palmer, R. D., Muschinski, A., Hooper, D. A., Schmidt, G., and Steinhagen, H.: SOMARE-99: A demonstrational field campaign for ultrahigh-resolution VHF atmospheric profiling using frequency diversity, Radio Sci., 36, 695-708, doi:10.1029/1999RS002308, 2001.

Chilson, P. B., Yu, T.-Y., Strauch, R. G., Muschinski, A., and Palmer, R. D.: Implementation of range imaging on the Platteville $915-\mathrm{MHz}$ tropospheric profiler, J. Atmos. Oceanic Technol., 20, 987-996, 2003.

Dalaudier, F., Sidi, C., Crochet, M., and Vernin, J.: Direct evidence of sheets in the atmospheric temperature field, J. Atmos. Sci., 51, 237-248, 1994.

Fernandez, J. R., Palmer, R. D., Chilson, P. B., Häggström, I., and Rietveld, M. T.: Range Imaging observations of PMSE using EISCAT VHF radar: Phase calibration and first results, Ann. Geophys., 23, 207-220, 2003.

Franke, S. J.: Pulse compression and frequency domain interferometry with a frequency-hopped MST radar, Radio Sci., 25, 565574, doi:10.1029/89RS03249, 1990.
Gavrilov, N. M., Luce, H., Crochet M., Dalaudier, F., and Fukao, S. Turbulence Parameter Estimations from High-Resolution Balloon Temperature Measurements of the MUTSI-2000 campaign, Ann. Geophys., 23, 2401-2413, 2005.

Kudeki, E. and Stitt, G. R.:Freqency domain interferometry: A high resolution radar technique for studies of atmospheric turbulence, Geophys. Res. Lett., 14, 1988-201, 1987.

Luce, H., Crochet, M., Hanuise, C., Yamamoto, M., and Fukao, S.: On the interpretation of the layered structures detected by mesosphere-stratosphere-troposphere radars in dual frequency domain interferometry mode, Radio Sci., 34, 1077-1084, doi:10.1029/1999RS900045, 1999.

Luce, H., Röttger, J., Crochet, M., Yamamoto, M., and Fukao, S.: Scattering layer thickness and position estimated by radar frequency domain interferometry, 2 Effects of tilts of the scattering layer or radar beam, Radio Sci., 35, 1109-1127, 2000.

Luce, H., Yamamoto, M., Fukao, S., Hélal, D., and Crochet, M.: A Frequency radar Interferometric Imaging applied with High Resolution Methods, J. Atmos. Sol. Terr. Phys., 63, 221-234, 2001a.

Luce, H., Yamamoto, M., Fukao, S., and Crochet, M.: Extended radar observations with the Frequency radar domain Interferometric Imaging (FII) technique, J. Atmos. Sol. Terr. Phys., 63, 1033-1041, 2001b.

Luce, H., Kubo, K., and Fukao, S.: An experimental contribution to the interpretation of mesosphere-stratosphere-troposphere radar echoes in frequency domain interferometric mode, Radio Sci., 36, 999-1012, doi:10.1029/2000RS002554, 2001c.

Muschinski, A. and Wode, C.: First in situ evidence for co-existing sub-meter temperature and humidity sheets in the lower free troposphere, J. Atmos. Sci., 55, 2893-2906, 1998.

Nickel, U.: Algebraic formulation of Kumaresan-Tufts superresolution method, showing relation to ME and MUSIC methods, IEE Proceedings, 135, 7-10, 1988.

Palmer, R. D., Yu, T-Y., and Chilson, P. B.: Range imaging using frequency diversity, Radio Sci., 34, 1485-1496, doi:10.1029/1999RS900089, 1999.

Palmer, R. D., Chilson, P. B., Muschinski, A., Schmidt, G., Yu, T.-Y., and Steinhagen, H.: SOMARE-99: Observations of tropospheric scattering layers using multiple-frequency range imaging, Radio Sci., 36, 681-694, doi:10.1029/1999RS002307, 2001.

Röttger, J. and Schmidt, G.: High-resolution VHF radar sounding of the troposphere and stratosphere, IEEE Trans. Geosci. Electron., 17, 182-189, 1979.

Smaïni, L., Luce, H., Crochet, M., and Fukao, S.: A new Highresolution method for Frequency domain Interferometric Imaging (FII) technique, J. Atmos. Oceanic. Tech., 19, 954-966, 2002.

Stoïca, P. and Moses, R.: Introduction to Spectral analysis, Prentice Hall, New Jersey, 1997.

Yu, T.-Y. and Brown, W. J.: High-resolution atmospheric profiling using combined spaced antenna and range imaging techniques, Radio Sci., 39, RS1011, doi:10.1029/2003RS002907, 2004.

Zhang, G., Yu, T.-Y., and Doviak R. J.: Angular and range interferometry to refine weather radar resolution, Radio Sci., 40, RS3013, doi:10.1029/2004RS003125, 2005. 